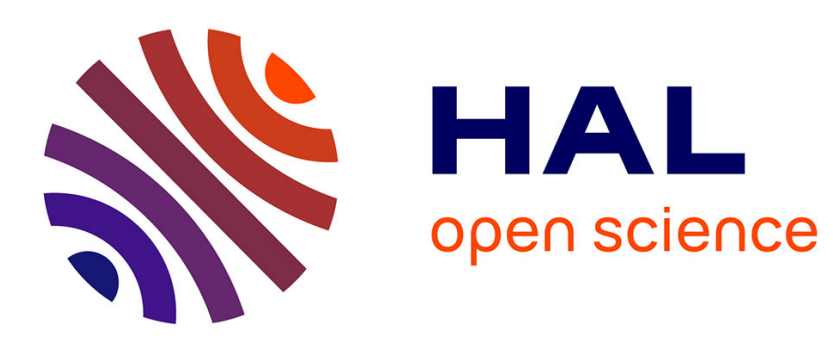

\title{
Accuracy of impact broadening models in low-density magnetized hydrogen plasmas
}

Joël Rosato, Hubert Capes, Laurence Godbert-Mouret, Mohammed Koubiti, Yannick Marandet, Roland Stamm

\section{- To cite this version:}

Joël Rosato, Hubert Capes, Laurence Godbert-Mouret, Mohammed Koubiti, Yannick Marandet, et al. Accuracy of impact broadening models in low-density magnetized hydrogen plasmas. Journal of Physics B: Atomic, Molecular and Optical Physics, 2012, Vol. 45, p.165701. hal-00945760

\section{HAL Id: hal-00945760 \\ https://hal.science/hal-00945760}

Submitted on 19 Feb 2014

HAL is a multi-disciplinary open access archive for the deposit and dissemination of scientific research documents, whether they are published or not. The documents may come from teaching and research institutions in France or abroad, or from public or private research centers.
L'archive ouverte pluridisciplinaire HAL, est destinée au dépôt et à la diffusion de documents scientifiques de niveau recherche, publiés ou non, émanant des établissements d'enseignement et de recherche français ou étrangers, des laboratoires publics ou privés. 


\title{
Accuracy of impact broadening models in low-density magnetized hydrogen plasmas
}

\author{
J Rosato, H Capes, L Godbert-Mouret, M Koubiti, Y \\ Marandet and R Stamm \\ Laboratoire PIIM, UMR 7345 Université d'Aix-Marseille / CNRS, Centre de \\ St-Jérôme, Case 232, F-13397 Marseille Cedex 20, France
}

\begin{abstract}
The impact approximation used in the modelling of Stark profiles is examined when a magnetic field is present. Motivated by tokamak plasma spectroscopy, we calculate line shapes and $S$-matrix elements for the first Lyman lines of hydrogen with two models proposed for retaining simultaneously Stark and Zeeman effects in the impact limit. An evaluation of the accuracy of the two approaches is made with the help of a numerical simulation.
\end{abstract}

\section{Introduction}

Detailed line shapes are of interest for accurate plasma diagnostics in magnetic fusion [1]. A renewal of interest in the modelling of Stark broadening has been provided recently in the context of radiation transport simulations for tokamak divertor physics. In divertors operating at high density regimes (up to $10^{15} \mathrm{~cm}^{-3}$ and higher: Alcator C-Mod [2] and soon ITER [3]), the plasma close to the wall contains a sufficiently large amount of neutrals so as to be optically thick to the first Lyman lines of the hydrogen isotopes. Coupled plasma-radiation-neutrals simulations have indicated the possibility for a shift of the ionization-recombination balance induced by the photoabsorption, in particular due to the Ly- $\alpha$ transition [4]. The need for reliability in these simulations has led to the development of accurate models for the spectral profiles which are involved in the photon emission and absorption rates appearing in the equation of radiative transfer. Efforts have been carried out in order to properly describe the ion dynamics on the Stark shape in the atoms' frame of reference [5]. The latter reference discusses in details the formation of Ly- $\alpha$ and includes the combined action of the Zeeman effect and the fine structure, which can be of the same order of magnitude (e.g. [6, 7] for early works).

This paper deals with Stark broadening at plasma conditions such that the impact approximation can be used for ions. In a recent work [8], a collision operator model accounting for the Zeeman degeneracy removal of the energy levels has been developed in the framework of the standard impact theory (Griem-Baranger-Kolb-Oertel or GBKOmodel [9]), hereafter referred to as ST. An application to the Lorentz triplet of Ly- $\alpha$ (strong field regime) has indicated the possibility for having the lateral components 
much narrower than the central component. In a work published last year $([10]$, note added in proof), it has been mentioned that the use of the ST leads to an overestimate of the "primary, adiabatic" and "secondary, nonadiabatic" contributions. Details have been published at the same time in [11]. We review in this paper the main features of the ST and of the alternative impact theory referred to as "generalized theory" (GT) in Refs. [10, 11]. The GT uses a different interaction representation than the ST and avoids the use of a cut-off for small impact parameters. In this work, we perform a detailed comparison between the two approaches, and discuss their respective accuracy in the treatment of strong collisions. We propose a way for a fair comparison between these approaches, and calculate the two resulting line shapes. Other comparisons are made for the $S$-matrix and line broadening cross section, for which the two models are also confronted to a numerical simulation enabling an evaluation of their accuracy. Since the GT is designed for parabolic states, our calculations neglect fine structure. Section 2 gives an introduction to the impact formalism, Sec. 3 and Sec. 4 present the ST and the GT, respectively, and Secs. 5, 6 report on comparisons with simulations. Details and clarifications for a meaningful comparison of the two approaches are given in the Appendix.

\section{Ion impact broadening: setting up the formalism}

The spectral profile of an atomic line, $I(\omega)$, is given by the Fourier transform of the atomic dipole autocorrelation function $C(t)$ :

$$
\begin{aligned}
& I(\omega)=\frac{1}{\pi} \operatorname{Re} \int_{0}^{+\infty} d t C(t) e^{i \omega t}, \\
& C(t)=\left\{\operatorname{Tr}\left(\rho_{u} \vec{d}_{\perp} \cdot U_{l}^{\dagger}(t) \vec{d}_{\perp} U_{u}(t)\right)\right\} .
\end{aligned}
$$

Here, $\vec{d}_{\perp}$ is the dipole operator projected into the polarization plane; $U(t)$ is the evolution operator; $\rho$ is the density operator; the brackets $\{\ldots\}$ stand for a statistical average over the perturbers' states and the trace $\operatorname{Tr}(\ldots)$ is performed over the atomic states. The subscripts $u$ and $l$ refer to the restriction of operators in the subspace corresponding to the upper and lower energy levels of the transition, respectively. For the sake of simplicity when examining the ST and the GT we will neglect the broadening of the lower levels (strictly speaking this is applicable to Lyman lines in the absence of fine structure and neglecting quenching interactions), so that $U_{l}(t)$ can be replaced by $1_{l}$, the identity matrix in the lower levels' subspace. A more general treatment, accounting for the lower levels, would consist in replacing all operators by super-operators acting in the Liouville "line" space [12]. Since all of the development done hereafter will exclusively concern the upper levels' subspace, we will not write the subscript $u$ anymore. For notational simplicity we will use SI-atomic units $\left(m_{e}=e=\hbar=a_{0}=1, \varepsilon_{0} \mu_{0} c^{2}=1\right.$, [13]).

Within the impact approximation for ions, the evolution operator is given by $U(t)=\exp \left[\left(-i H_{0}-\Phi\right) t\right]$ where $H_{0}$ is the atomic Hamiltonian accounting for the Zeeman 
effect, i.e. $H_{0}=H_{\mathrm{at}}-\vec{\mu} \cdot \vec{B}$ with $\vec{\mu}, \vec{B}$ being respectively the magnetic moment and the magnetic field; and $\Phi$ is the ion collision operator $\{$ the electron contribution is neglected since weaker by a factor $\left(2 m_{e} / m_{i}\right)^{1 / 2}$ at equal temperatures, $m_{e}, m_{i}$ being the electron and ion masses, respectively [8]\}. Its matrix elements denote typical collision frequencies and provide the width and the shift of the line under consideration, of Lorentzian shape. This operator is defined by

$$
\Phi=N \int_{0}^{+\infty} d v f(v) v \int_{0}^{\lambda_{D}} d b 2 \pi b\{1-S(\vec{b}, \vec{v})\}_{\text {angle }},
$$

where the integrals are done over the velocity (module) $v$ and the impact parameter (module) $b, N$ and $f$ stand for the perturbers' density and velocity distribution function, the brackets denote an angular average and $S$ is the scattering matrix corresponding to a binary collision. The emitter motion is accounted for through a reduced mass model. The upper bound $\lambda_{D}$ is the Debye length, which accounts phenomenologically for the screening of the Coulomb field due to the electrons. In the following we consider the cross section operator $\sigma(v)=\int_{0}^{\lambda_{D}} d b 2 \pi b\{1-S(\vec{b}, \vec{v})\}_{\text {angle }}$ and its matrix elements $\left\langle\alpha|\sigma(v)| \alpha^{\prime}\right\rangle \equiv \sigma_{\alpha \alpha^{\prime}}$

\section{Standard impact theory}

In the standard theory (ST) [9], the $S$-matrix is calculated in the interaction picture defined by the unitary transformation $|\psi(t)\rangle \rightarrow|\tilde{\psi}(t)\rangle=\exp \left(i H_{0} t\right)|\psi(t)\rangle$. This leads to a time-ordered exponential

$$
\begin{aligned}
S & =\mathcal{T} \exp \left[-i \int_{-\infty}^{+\infty} d t V(t)\right] \\
& =1-i \int_{-\infty}^{+\infty} d t V(t)-\int_{-\infty}^{+\infty} d t V(t) \int_{-\infty}^{t} d t^{\prime} V\left(t^{\prime}\right)+\ldots
\end{aligned}
$$

where $V(t)=-\tilde{\vec{d}}(t) \cdot \vec{E}(t)$ is the Stark term in the interaction picture corresponding to a binary interaction with an ion [i.e. $\vec{E}(t)=-(\vec{b}+\vec{v} t) /\left(b^{2}+v^{2} t^{2}\right)^{3 / 2}$ ]. Following [9], we separate the $b$-integral in Eq. (3) into two parts, which correspond to two regimes. (i) Weak collisions: The time-integrated perturbation is sufficiently small so that a second order approximation can be used. A condition on $(\vec{b}, \vec{v})$, which ensures the validity of this approximation, may be formulated as $\left|\left\{S_{\alpha \alpha}^{(2)}\right\}_{\text {angle }}\right| \leq 1$, where $S^{(2)}$ stands for the second order term of the $S$-matrix (with the convention $S=S^{(0)}+S^{(1)}+S^{(2)}+\ldots$ ) and $\alpha$ refers to the state $|\alpha\rangle$. (ii) Strong collisions: When the second order approximation cannot be applied, the $S$-matrix becomes a strongly oscillating function of $b$, so that its integral over $b$ can be neglected compared to the integral of the identity matrix (such a treatment is appropriate in particular when the contribution due to weak collisions is dominant, e.g. [14] for a detailed discussion). For each state $|\alpha\rangle$, the two regimes (i) and (ii) correspond to impact parameters large and small compared to the strong collision radius $b_{\alpha}^{\text {st }}$, respectively. The latter is defined by the following transcendental equation 
[8]

$$
b_{\alpha}^{\mathrm{st}}=b_{W \alpha}\left\{\left[K_{\alpha}^{/ /}+K_{\alpha}^{\perp} A_{\mathrm{ST}}\left(\frac{b_{\alpha}^{\mathrm{st}}}{b_{m}}\right)\right]^{2}+\left[K_{\alpha}^{\perp} B_{\mathrm{ST}}\left(\frac{b_{\alpha}^{\mathrm{st}}}{b_{m}}\right)\right]^{2}\right\}^{1 / 4},
$$

where $b_{W \alpha}=(2 / 3)^{1 / 2} n_{\alpha}^{2} / v$ is the Weisskopf radius ( $n_{\alpha}$ is the principal quantum number); $b_{m}=2 v / B$ is the magnetic cut-off; $K_{\alpha}^{/ /}, K_{\alpha}^{\perp}, K_{\alpha}^{\perp \perp}$ are atomic constants defined by

$$
\begin{aligned}
K_{\alpha}^{/ /} & =\frac{1}{n_{\alpha}^{4}} \sum_{\alpha^{\prime}}\left|z_{\alpha^{\prime} \alpha}\right|^{2}, \\
K_{\alpha}^{\perp} & =\frac{1}{n_{\alpha}^{4}} \sum_{\alpha^{\prime}}\left[\left|x_{\alpha^{\prime} \alpha}^{+}\right|^{2}+\left|x_{\alpha^{\prime} \alpha}^{-}\right|^{2}\right], \\
K_{\alpha}^{\prime \perp} & =\frac{1}{n_{\alpha}^{4}} \sum_{\alpha^{\prime}}\left[\left|x_{\alpha^{\prime} \alpha}^{+}\right|^{2}-\left|x_{\alpha^{\prime} \alpha}^{-}\right|^{2}\right],
\end{aligned}
$$

with the convention $x^{ \pm}=(x \pm i y) / \sqrt{2}$; and $A_{\mathrm{ST}}(s)=s^{2}\left[K_{0}^{2}(s)+K_{1}^{2}(s)\right]$ and $B_{\mathrm{ST}}(s)=$ $s^{2}\left[K_{0}(s) I_{0}(s)-K_{1}(s) I_{1}(s)\right]$ with $I_{0,1}$ and $K_{0,1}$ being the modified Bessel functions.

In the following $b_{\alpha}^{\text {st }} \leq \lambda_{D}$ will be assumed. We can write the diagonal matrix elements of the cross section operator as follows:

$$
\begin{aligned}
\sigma_{\alpha \alpha} & =\int_{0}^{b_{\alpha}^{\text {st }}} d b 2 \pi b+\int_{b_{\alpha}^{\text {st }}}^{\lambda_{D}} d b 2 \pi b\left\{1-S_{\alpha \alpha}^{(0)}(\vec{b}, \vec{v})-S_{\alpha \alpha}^{(1)}(\vec{b}, \vec{v})-S_{\alpha \alpha}^{(2)}(\vec{b}, \vec{v})\right\}_{\text {angle }} \\
& =\pi\left(b_{\alpha}^{\text {st }}\right)^{2}-\int_{b_{\alpha}^{\text {st }}}^{\lambda_{D}} d b 2 \pi b\left\{S_{\alpha \alpha}^{(2)}(\vec{b}, \vec{v})\right\}_{\text {angle }} .
\end{aligned}
$$

Here we have used $S^{(0)}=1$ and $\left\{S^{(1)}\right\}_{\text {angle }}=0$ (isotropic medium). The angle average and the integral over $b$ present in the second term of the right-hand side can be performed analytically. An explicit calculation leads to [8]

$$
\sigma_{\alpha \alpha}=\pi\left(b_{\alpha}^{\mathrm{st}}\right)^{2}+2 \pi b_{W \alpha}^{2}\left\{K_{\alpha}^{/ /} \ln \left(\frac{\lambda_{D}}{b_{\alpha}^{\mathrm{st}}}\right)+\left[K_{\alpha}^{\perp} a_{\mathrm{ST}}(s)-i K_{\alpha}^{\prime \perp} b_{\mathrm{ST}}(s)\right]_{s=\lambda_{D} / b_{m}}^{s=b_{\alpha}^{\mathrm{st}} / b_{m}}\right\}
$$

where $a_{\mathrm{ST}}(s)=s K_{0}(s) K_{1}(s)$ and $b_{\mathrm{ST}}(s)=\pi / 2-\pi s K_{0}(s) I_{1}(s)$ are the GBKO functions $(s>0)$. In accordance with the model, there is a separation between the strong collisions (first term of the right-hand side) and the weak collisions (second term). The strong collisions are described by a Lorentz-Weisskopf term of characteristic radius $b_{\alpha}^{\text {st }}$. The latter is calculated from Eq. (5). It accounts for the Zeeman degeneracy removal and reduces to $b_{W \alpha}$ (up to a factor dependent on the quantum numbers) when $B \rightarrow 0$. The weak collision term is split into two parts, which correspond to the electric field component parallel $(/ /)$ and perpendicular $(\perp)$ to $\vec{B}$. The magnetic field leads to a reduction of the perpendicular contribution. In the particular case where $b_{\alpha}^{\text {st }} \ll b_{m} \ll \lambda_{D},\left.a_{\mathrm{ST}}(s)\right|_{s=\lambda_{D} / b_{m}} ^{s=b_{\alpha}^{\mathrm{st}} / b_{m}}$ behaves as $\ln \left(b_{m} / b^{\mathrm{st}}\right)$, indicating that the effective range of the perpendicular electric field is reduced from $\lambda_{D}$ to $b_{m}$ (see [8] for an interpretation with classical mechanics). In practice, such a reduction leads to a narrowing of the Zeeman components. As a particular example consider the lateral components of the Zeeman-Lorentz triplet of hydrogen Ly- $\alpha$, which are broadened due to the perpendicular contribution only. Figure 1 shows the blue component calculated assuming degenerate 


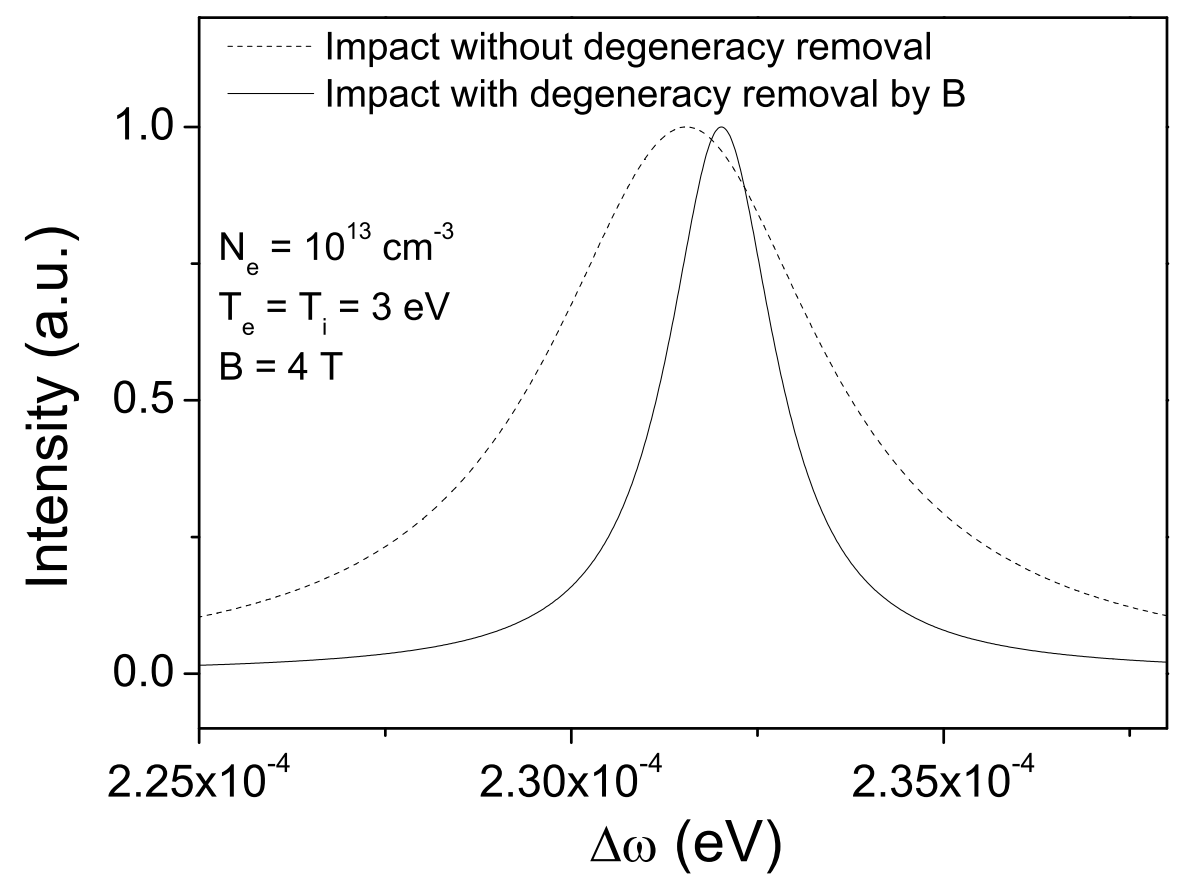

Figure 1. Blue lateral component of the Zeeman-Lorentz triplet of the Lyman- $\alpha$ line, obtained using the standard impact theory neglecting (dashed line) and retaining the degeneracy removal (solid line). A strong reduction of the line width, by more than a factor of two, is present. A shift is also visible.

levels [i.e. formally setting $b_{m} \rightarrow \infty$ in Eqs. (5), (10)] and assuming finite $b_{m}$, at $N_{e}=10^{13} \mathrm{~cm}^{-3}, T_{e}=T_{i}=3 \mathrm{eV}$, and $B=4 \mathrm{~T}$. As can be seen, the model predicts a component much narrower (by more than a factor of two) than expected when neglecting the Zeeman degeneracy removal. The component is also shifted to larger frequencies [this stems from the presence of an imaginary part in Eq. (10)].

\section{Generalized theory}

The GT is based on an interaction picture different from that introduced above. We follow the synthesis presented in [15] (details on the derivation of formulas can be found in [16]). All matrix elements refer to the parabolic base. The unitary transformation is done by including the contribution of the electric field parallel to $\vec{B}$ in the unperturbed Hamiltonian, namely $|\psi(t)\rangle \rightarrow|\tilde{\psi}(t)\rangle=\exp \left[i H_{0} t-i \int_{-\infty}^{t} d t^{\prime} d_{/ /} E_{/ /}\left(t^{\prime}\right)\right]|\psi(t)\rangle \equiv$ $Q^{\dagger}(t)|\psi(t)\rangle$. This leads to the following expression for the $S$-matrix

$$
\begin{aligned}
& S=S_{a} \mathcal{T} \exp \left[i \int_{-\infty}^{+\infty} d t Q^{\dagger}(t) \vec{d}_{\perp} \cdot \vec{E}_{\perp}(t) Q(t)\right], \\
& S_{a}=\exp \left[i \int_{-\infty}^{+\infty} d t d_{/ /} E_{/ /}(t)\right] .
\end{aligned}
$$


The quantity $S_{a}$ denotes the solution that would be obtained in the $B=0$-case if timeordering was neglected (adiabatic approximation). If $B$ is finite, $S_{a}$ corresponds to the solution that would be obtained if the virtual transitions between the Zeeman energy sublevels (i.e., the inelastic collisions) were neglected. Using Eqs. (11) and (12), $S$ can be decomposed into $S_{a}+S_{\text {na }}$, where the non-adiabatic contribution $S_{\text {na }}$ is given by

$$
S_{\text {na }}=S_{a}\left\{\mathcal{T} \exp \left[i \int_{-\infty}^{+\infty} d t Q^{\dagger}(t) \vec{d}_{\perp} \cdot \vec{E}_{\perp}(t) Q(t)\right]-1\right\}
$$

Accordingly, the cross section operator also splits into two parts $\sigma=\sigma^{a}+\sigma^{\text {na }}$. In the GT, $S_{a}$ is calculated exactly and a second-order approximation is done concerning $S_{\text {na }}$. No distinction is made between the weak and strong collisions, i.e., the integral over $b$ is carried out from 0 to $\lambda_{D}$ even though the second-order expansion is not valid for small values of $b$. In $[15,16]$, this procedure is justified by the fact that the integral over $b$ converges without the need for a cut-off. Physically, this convergence is due to the dressing of the energy levels by $E_{/ /}(t)$. Collisions with small impact parameters lead to a strong degeneracy removal of the energy levels, so that the effect of $\vec{E}_{\perp}$ is reduced in such a way that the cross section remains finite. However, this does not guarantee that the second-order approximation provides a reliable estimate of $S_{\text {na }}$, since the contribution of higher order terms is in principle not negligible. We will see in the next section that ignoring these higher order terms leads to overestimate the strong collision contribution and, eventually, the line width.

The adiabatic cross section operator is given by

$$
\sigma^{a}=\int_{0}^{\lambda_{D}} d b 2 \pi b\left\{1-S^{a}\right\}_{\text {angle }}
$$

It is diagonal in the parabolic base. The corresponding cross section reads

$$
\begin{aligned}
& \sigma^{a}=2 \pi\left(\frac{2 z_{\alpha \alpha}}{v}\right)^{2} I\left(\frac{v \lambda_{D}}{2 z_{\alpha \alpha}}\right), \\
& I(s)=\frac{1}{6}\left[3 s^{2}-2 s^{3} \sin \left(\frac{1}{s}\right)-s^{2} \cos \left(\frac{1}{s}\right)+s \sin \left(\frac{1}{s}\right)-\mathrm{Ci}\left(\frac{1}{s}\right)\right],
\end{aligned}
$$

where $\mathrm{Ci}$ is the cosine integral function. $I(s)$ has the following asymptotic behavior for large $s$ :

$$
I(s) \sim \frac{11}{36}-\frac{\gamma}{6}+\frac{1}{6} \ln s,
$$

where $\gamma \simeq 0.577$ is Euler's constant. In this expression the logarithm corresponds to the weak collisions.

The non-adiabatic cross section operator is given by

$$
\sigma^{\mathrm{na}}=-\int_{0}^{\lambda_{D}} d b 2 \pi b\left\{S^{\mathrm{na}}\right\}_{\text {angle }}
$$

and the corresponding cross section reads

$$
\sigma_{\alpha \alpha^{\prime}}^{\mathrm{na}}=\frac{4 \pi}{3 v^{2}} \sum_{\alpha^{\prime \prime}}\left[x_{\alpha \alpha^{\prime \prime}}^{+} x_{\alpha^{\prime \prime} \alpha^{\prime}}^{-}+x_{\alpha \alpha^{\prime \prime}}^{-} x_{\alpha^{\prime \prime} \alpha^{\prime}}^{+}\right] \int_{0}^{\lambda_{D} / b_{m}} \frac{d s}{s} C_{ \pm}(\chi, Y, s),
$$


where $\chi=z_{\alpha \alpha} / \delta z$ with $\delta z=\left(z_{\alpha \alpha}-z_{\alpha^{\prime \prime} \alpha^{\prime \prime}}\right)$, and $Y=\delta z / v b_{m}$. In the integral, $C_{ \pm}$is the so-called broadening function, given by [15]

$$
\begin{aligned}
& C_{ \pm}(\chi, Y, s)=\frac{3}{4} \int_{-\infty}^{\infty} d x \int_{-\infty}^{x} d x^{\prime}\left[w(x) w\left(x^{\prime}\right)\right]^{3} e^{i s\left(x-x^{\prime}\right)} \\
& \quad \times\left\{j_{0}(\varepsilon)+\left(2 x x^{\prime}-1\right) \frac{j_{1}(\varepsilon)}{\varepsilon}+\left[\left(1-x x^{\prime}\right) \sigma_{1}^{2}-\left(x+x^{\prime}\right) \sigma_{1} \sigma_{2}\right] \frac{j_{2}(\varepsilon)}{\varepsilon^{2}}\right\} \\
& \varepsilon=\sqrt{\sigma_{1}^{2}+\sigma_{2}^{2}} \\
& \sigma_{1}=\frac{Y}{s}\left[x w(x) \pm x^{\prime} w\left(x^{\prime}\right)+1 \pm 1-2 \chi\right] \\
& \sigma_{2}=\frac{Y}{s}\left[w(x) \pm w\left(x^{\prime}\right) \chi\right] \\
& w(x)=\left(1+x^{2}\right)^{-1 / 2}
\end{aligned}
$$

Here $j_{0}, j_{1}, j_{2}$ denote the first spherical Bessel functions. The upper signs correspond to the nondiagonal matrix elements $\left(\alpha^{\prime} \neq \alpha\right)$ and the lower signs correspond to the diagonal matrix elements $\left(\alpha^{\prime}=\alpha\right)$. In the limiting case $B \rightarrow 0, C_{ \pm}$reduces to $A_{\mathrm{ST}}+i B_{\mathrm{ST}}$, the integral Eq. (19) diverges at the lower bound $s=0$, and the upper bound tends to 0 . In this case, a cut-off denoting separation between the weak and strong collisions (as in the ST) is used. At finite $B$, the virtual transitions between the Zeeman sublevels mitigate the role of the non-adiabatic contribution. This leads to a narrowing of the line components. This point is illustrated in Fig. 2. The blue lateral component of Ly- $\alpha$ obtained within the GT is superimposed to the result obtained at $B=0$, at the same conditions as in the previous section. In this particular case, the line broadening is given by the non-adiabatic contribution only. As can be seen, the model predicts a component narrower than expected when neglecting the Zeeman degeneracy removal and the component is shifted to larger frequencies. The width reduction is weaker than that predicted with the ST, with only $40 \%$ instead of a factor larger than two.

\section{Comparison of the two models to a simulation}

We have compared the GT and ST to the result of a fully numerical calculation of the collision operator. In this method, the $S$-matrix is calculated for a set of impact parameters $\vec{b}$ and velocities $\vec{v}$ chosen in such a way that the perturber follows a straight line in the Debye sphere surrounding the atom, in agreement with the straight line trajectory assumption done both within the GT and the ST. The algorithm used for the integration of the Schrödinger equation uses the infinitesimal evolution operator $U(t+\Delta t, t)$, i.e. we evaluate $S \simeq U(T / 2,-T / 2)$ for large $T$ by iterating the relation $U(t+\Delta t,-T / 2)=U(t+\Delta t, t) U(t,-T / 2)$. The infinitesimal evolution operator is approximated as $U(t+\Delta t, t) \simeq \exp [-i \Delta t V(t)]$, taking $\Delta t \ll$ $\sqrt{b^{2}+v^{2} t^{2}} / v, 1 / \omega_{S}, 1 / \omega_{Z}$ (with $\omega_{S}, \omega_{Z}$ being the typical Stark and Zeeman frequencies estimated from matrix elements of $-\vec{d} \cdot \vec{E}(t)$ and $-\vec{\mu} \cdot \vec{B}$, respectively). The matrix 


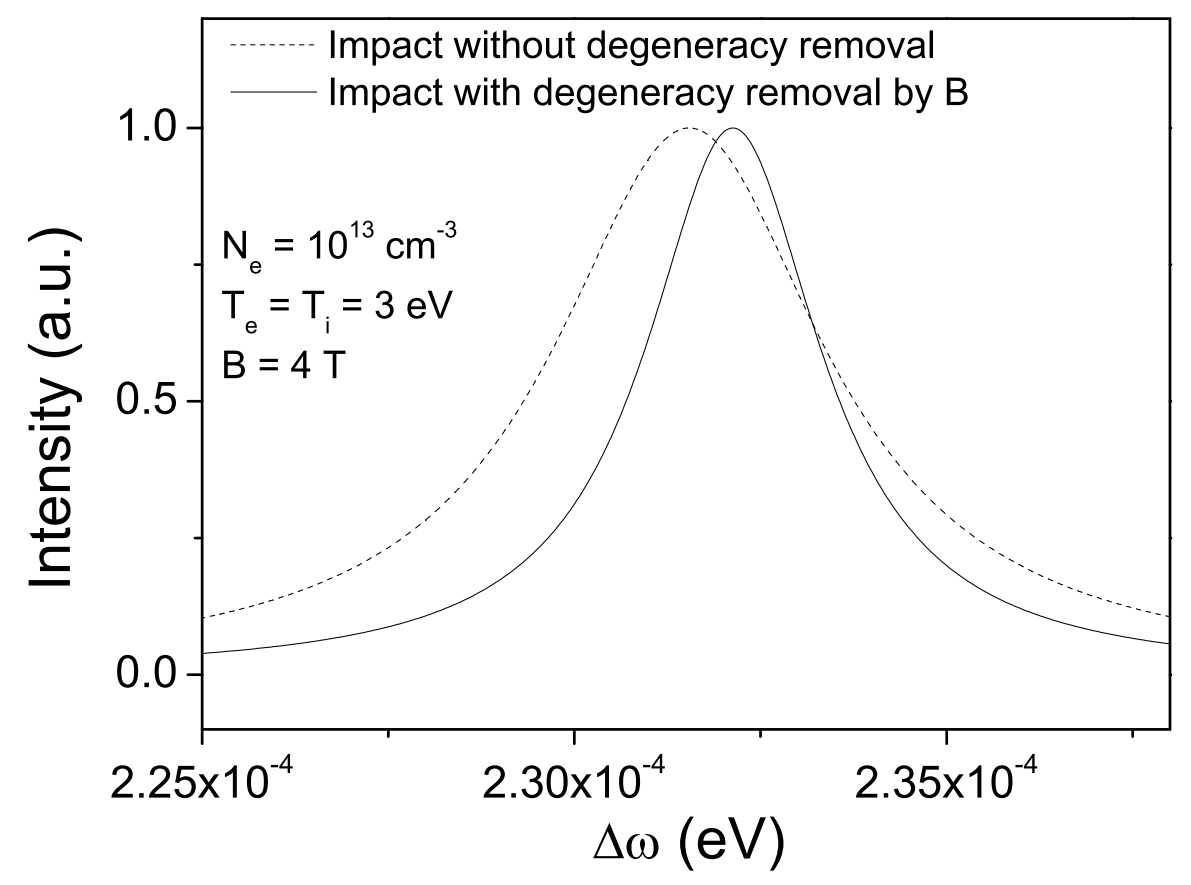

Figure 2. The non-adiabatic contribution is mitigated by the Zeeman degeneracy removal, which leads to line narrowing. Here, plot of the blue lateral component of Lyman- $\alpha$ obtained using the generalized theory neglecting (dashed line) and retaining the degeneracy removal (solid line). As in the previous section, a shift is also visible.

exponential is evaluated using the scaling-squaring method, i.e. we use the identity $\exp [-i \Delta t V(t)] \equiv\{\exp [-i \Delta t V(t) / m]\}^{m}$ with $m$ integer and we choose $m$ sufficiently large so that the matrix elements of $\Delta t V(t) / m$ are small in absolute value (details on the scaling-squaring method can be found in [17] and Refs. therein). Then, the exponential is approximated by a low-order expansion. The angle average is performed by MonteCarlo integration. We have examined the matrix element $\left\langle 211\left|\{S(\vec{b}, \vec{v})\}_{\text {angle }}\right| 211\right\rangle$, which is affected by the non-adiabatic contribution exclusively (here a notation in terms of the spherical base quantum numbers is used). Figure 3 shows a plot of the real and imaginary part in terms of $b$ (atomic units), assuming $v=\sqrt{2 T_{i} / m_{i}}$, at $N_{e}=10^{13} \mathrm{~cm}^{-3}$, $T_{e}=T_{i}=3 \mathrm{eV}$ and $B=4 \mathrm{~T}$, obtained from the numerical calculation and compared to the ST and the GT. The broadening function involved in the GT has been evaluated following Eq. (20). Here, $\chi=0$. The double integral has been calculated by MonteCarlo method, interpreting $\left[w(x) w\left(x^{\prime}\right)\right]^{3}$ as a joint probability density function for $x, x^{\prime}$. As can be seen, both the ST and the GT agree well with the numerical result at impact parameters larger than $\sim 200$, which roughly corresponds to the strong collision radius $b_{\text {st }}$ as defined in Eq. (5). Below this radius the numerical solution oscillates around zero, with an increasing frequency as $b$ decreases. This reinforces the choice of a cut-off made by the ST as described in Sec. 2. The GT yields a solution much different from the 

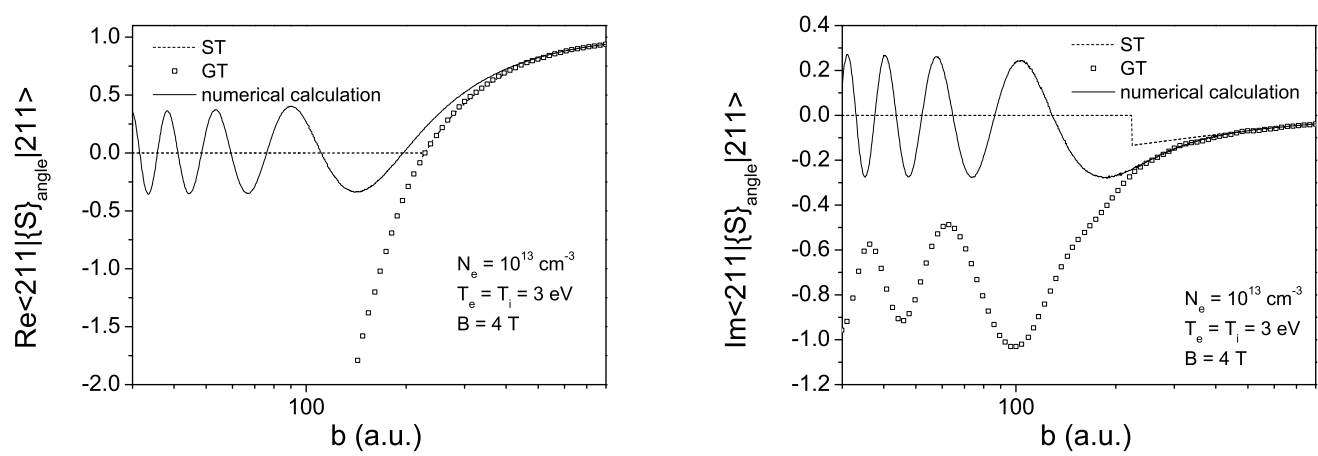

Figure 3. Real and imaginary parts of $\left\langle 211\left|\{S(\vec{b}, \vec{v})\}_{\text {angle }}\right| 211\right\rangle$, obtained within the ST and the GT, and compared to a simulation. For impact parameters larger than the strong collision radius $b_{\mathrm{st}}$ (of the order of 200), the ST and the GT agree well with the numerical calculation. The presence of oscillations at impact parameters smaller than $b_{\text {st }}$ corresponds to strong collisions which, within the ST, are accounted for by a cut-off procedure. In this domain the GT overestimates the real and imaginary parts in absolute value.
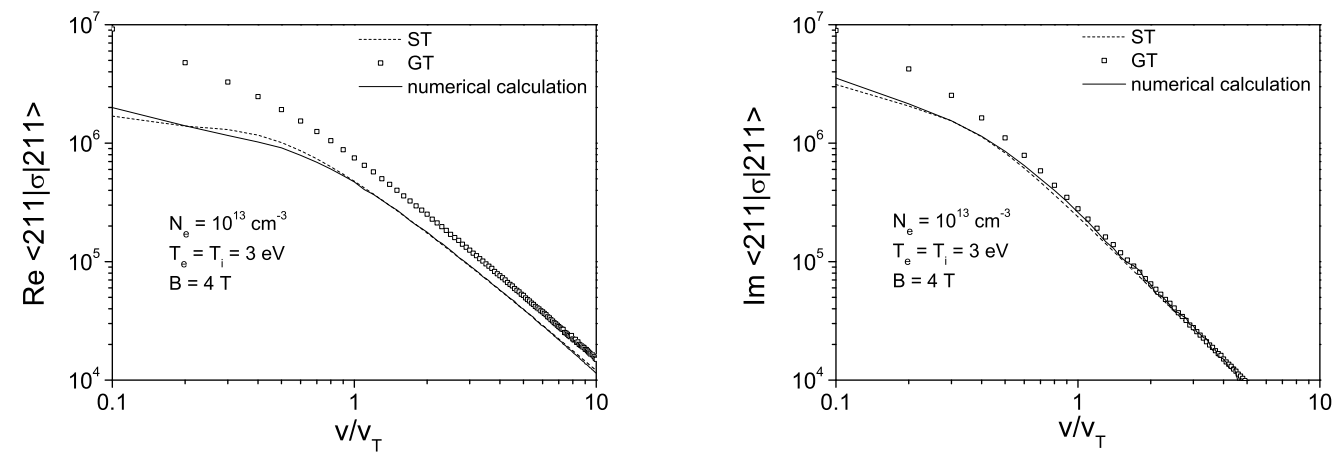

Figure 4. Real and imaginary parts of $\langle 211|\sigma| 211\rangle$, obtained within the ST and the GT, and compared to the simulation. $v_{T}=\sqrt{4 T_{i} / m_{i}}$ stands for the relative thermal velocity. The GT systematically overestimates the cross section, which results in an overestimate of the Stark width and shift.

numerical one, with a strong overestimate of the real and imaginary parts in absolute value (in particular the real part obtained with the GT diverges when $b \rightarrow 0$ ). This may lead to an overestimate of the Stark width and shift. This point is illustrated in Fig. 4 , where plots of the real and imaginary parts of the cross section are presented. The result of the GT is always larger than that of the numerical calculation. A calculation of the integral Eq. (3) shows that the GT overestimates the line width and shift by $60 \%$ and $20 \%$, respectively, whereas the ST never deviates from the simulation by more than $10 \%$. 

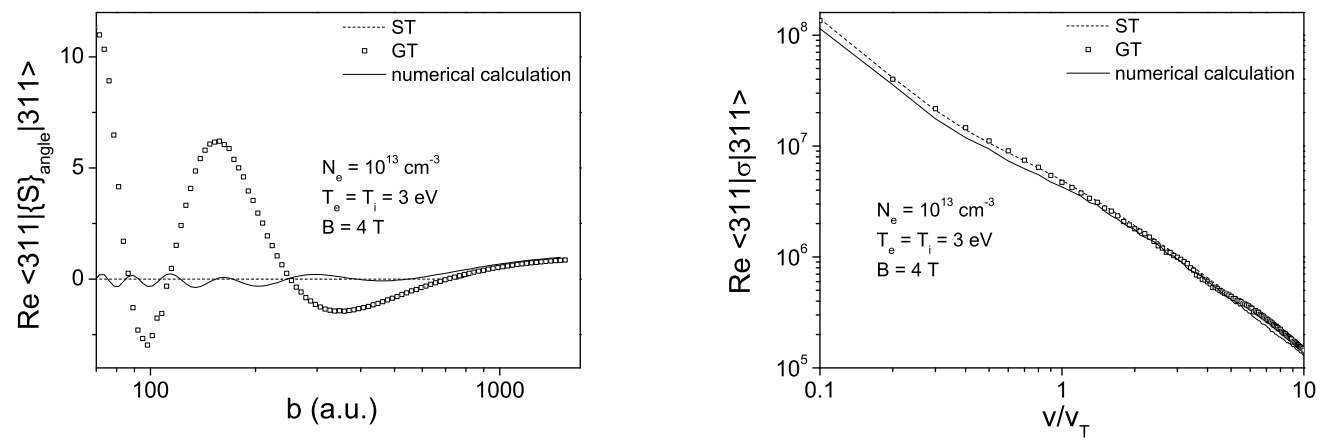

Figure 5. Real part of $\left\langle 311\left|\{S(\vec{b}, \vec{v})\}_{\text {angle }}\right| 311\right\rangle$ and the corresponding cross section, obtained within the ST and the GT, and compared to a simulation. The $S$-matrix obtained within the GT is not unitary at small impact parameters. The cross section is not overestimated here because of the presence of oscillations.

\section{States with permanent dipoles}

The particular case $\chi=0$ considered above denotes broadening of a parabolic state with spherical symmetry (i.e., $z_{\alpha \alpha}=0$ in the parabolic base). For hydrogen lines other than Ly- $\alpha$, the non-adiabatic contribution also involves states with a permanent dipole moment. We have examined such states by confronting the ST and the GT to simulations. Figure 5 shows a plot of the real part of $\left\langle 311\left|\{S(\vec{b}, \vec{v})\}_{\text {angle }}\right| 311\right\rangle$ and the corresponding cross section at the same conditions as in the previous section. This case involves the parabolic kets $|011\rangle$ and $|101\rangle$, i.e. states with a permanent dipole moment (e.g. [18] for details on the parabolic states). As can be seen, the GT yields oscillations at small impact parameters, with increasing amplitude as $b$ decreases. Although the $S$-matrix resulting from the GT is no longer unitary in this regime, the presence of oscillations prevents from the overestimation discussed above and eventually leads to a cross section similar to that obtained either within the ST or the numerical calculation. In general, the spherical states contribute to the broadening in addition to those with a permanent dipole, so that serious misestimates of the line width should still be expected a priori when using the GT. For example, an application to the blue lateral component of H- $\alpha$ (which involves the spherical state $|322\rangle$ ) shows that the GT already overestimates the width obtained by simulation by $70 \%$ when the broadening of the lower level is neglected, whereas deviations no larger than $10 \%$ are obtained with the ST.

\section{Conclusion}

The work presented in this paper provides a complement to previous investigations in Stark profile modelling for magnetic fusion plasmas. Two models for ion impact broadening - namely, the standard theory (ST) and the generalized theory (GT) - have been examined and compared to simulations. In general, the ST provides accurate 
results whereas the GT systematically overestimates the width and the shift of the Zeeman components because of an inconsistent use of the perturbation theory involved in the non-adiabatic part of the collision operator. In particular, a second-order expansion is used for the strong collisions, i.e., precisely, where a perturbative treatment does not apply. Although such collisions are not frequent (since $b_{\text {st }} \ll r_{0}$ is assumed in the impact theory, $r_{0}$ being the mean interparticle distance), the failure of the perturbation theory yields a sufficiently large error so as to globally affect the line shape. This suggests a subsequent refinement of this theory, either based on a cut-off procedure or on an analytical extension of the Dyson series expansion up to higher orders. Our conclusion is in agreement with previous studies regarding the GT at zero magnetic field [19]. The broadening function used in the GT involves a multiple integral of strongly oscillating functions, not suitable for fast numerical evaluation. A further refinement of the GT should consist in establishing a simple, CPU-convenient approximation for this integral. In magnetic fusion plasmas, the typical densities are sufficiently low so that the fine structure can be in competition both with the Stark and Zeeman effects. Another refinement of the GT should also address this issue. For opacity calculation purposes, accurate and analytical expressions are required for line shapes, so that the ST (and derived approaches accounting for non-impact effects, e.g., the unified theory [20, 21]) presently seems to be the best approach.

\section{Acknowledgments}

This work is supported by the collaboration LRC DSM99-14 between the PIIM laboratory and the CEA Cadarache research center, within the French federation of research on magnetic confinement fusion (FRFCM).

\section{Appendix}

In $[10,11]$, it is mentioned that the ST overestimates the adiabatic contribution "by up to two orders of magnitude". We have examined the relevance of this statement. The largest deviation claimed in [11] concerns the (102) - (101) component of the Paschen$\alpha$ line (parabolic numbers), hence here we focus our analysis on this transition. The calculation of the ratio $\sigma_{\alpha \alpha}^{\mathrm{ST}} / \sigma_{\alpha \alpha}^{\mathrm{GT}, a}$ in Ref. [11] is inconclusive for two reasons: (i) Both the broadening of the lower level and interference effects have been retained in $\sigma_{\alpha \alpha}^{\mathrm{GT}, a}\{\mathrm{cf}$. the definition of $X_{\alpha \beta}$ in [11], Eq. (A.1) $\}$ whereas not in $\sigma_{\alpha \alpha}^{\text {ST }}$. These effects, absent on Lyman lines, become important as the lower principal quantum number of the transition increases. A meaningful comparison between the two theories should involve, or not, the broadening of the lower level and the interference effects, at the same time. In the presentation of the ST done above as well as in that reported in [8], we have not retained the interference effects, but they can be easily treated within the Liouville line space formalism. (ii) $K_{\alpha}^{/ /} \equiv 1$ has been used for calculating the ST formulas. This is a very drastic approximation. For the upper level of the transition, $n=4, n_{1}=1, n_{2}=0$, 
$m=2$, so that $K_{\alpha}^{/ /}=9 / 64 \simeq 0.14$. A straightforward use of the formulas given by Eqs. (10) and (15) shows that

$$
\begin{aligned}
& \sigma_{\alpha \alpha}^{\mathrm{ST}}=\frac{32 \pi}{v^{2}}\left[\frac{3}{16}+\ln \left(\frac{v \lambda_{D}}{2 \sqrt{6}}\right)\right], \\
& \sigma_{\alpha \alpha}^{\mathrm{GT}, a}=\frac{48 \pi}{v^{2}}\left[\frac{11}{6}-\gamma+\ln \left(\frac{v \lambda_{D}}{12}\right)\right],
\end{aligned}
$$

so that, at $N_{e}=10^{13} \mathrm{~cm}^{-3}$ and $T_{e}=T_{i}=4 \mathrm{eV}$ (plasma conditions assumed in [11]), one has $\sigma_{\alpha \alpha}^{\mathrm{ST}} / \sigma_{\alpha \alpha}^{\mathrm{GT}, a} \simeq 0.65$. The magnitude of this deviation falls in the typical uncertainty range expected by the use of the GT and presented in the present paper.

\section{References}

[1] Goto M, Sakamoto R and Morita S 2007 Plasma Phys. Control. Fusion 49 1163-1176

[2] Welch B L, Griem H R, Terry J, Kurz C, LaBombard B, Lipschultz B, Marmar E and McCracken G 1995 Phys. Plasmas 2 4246-51

[3] Rosato J, Kotov V and Reiter D 2010 J. Phys. B: At. Mol. Opt. Phys. 43 144024-1-7

[4] Kotov V, Reiter D, Kukushkin A S, Pacher H D, Börner P and Wiesen S 2006 Contrib. Plasma Phys. 46 635-42

[5] Rosato J, Reiter D, Kotov V, Marandet Y, Capes H, Godbert-Mouret L, Koubiti M and Stamm R 2010 Contrib. Plasma Phys. 50 398-403

[6] Stehlé C and Feautrier N 1985 J. Phys. B: At. Mol. Phys. 18 1297-1306

[7] Stehlé C, Brillant S and Mathys G 2000 Eur. Phys. J. D 11 491-503

[8] Rosato J, Marandet Y, Capes H, Ferri S, Mossé C, Godbert-Mouret L, Koubiti M and Stamm R 2009 Phys. Rev. E 79 046408-1-7

[9] Griem H R, Baranger M, Kolb A C and Oertel G 1962 Phys. Rev. 125 177-195

[10] Oks E 2011 J. Phys. B: At. Mol. Opt. Phys. 44 101004-1-4

[11] Oks E 2010 International Review of Atomic and Molecular Physics 1 169-179

[12] Fano U 1963 Phys. Rev. 131 259-268

[13] Bransden B H and Joachain C J 1983 Physics of Atoms and Molecules (New York: Longman)

[14] Alexiou S 1995 Phys. Rev. Lett. 75 3406-3409

[15] Derevianko A and Oks E 1994 Phys. Rev. Lett. 73 2059-2062

[16] Ispolatov Ya and Oks E 1994 J. Quant. Spectrosc. Radiat. Transfer 51 129-138

[17] Moler C and Van Loan C 2003 SIAM Rev. 45 3-49

[18] Bethe H A and Salpeter E E 1957 Quantum Mechanics of One- and Two-Electron Atoms (Berlin: Springer)

[19] Alexiou S, Griem H R, Halenka J and Olchawa W 2006 J. Quant. Spectrosc. Radiat. Transfer 99 $238-251$

[20] Voslamber D 1969 Z. Naturforsch. 24a 1458-72

[21] Smith E W, Cooper J and Vidal C R 1969 Phys. Rev. 185 140-51 